\title{
On a bound of Cocke and Venkataraman
}

\author{
Benjamin Sambale ${ }^{1}(\mathbb{D}) \cdot$ Philipp Wellmann $^{1}$
}

Received: 4 May 2021 / Accepted: 10 June 2021 / Published online: 1 July 2021

(C) The Author(s) 2021

\begin{abstract}
Let $G$ be a finite group with exactly $k$ elements of largest possible order $m$. Let $q(m)$ be the product of $\operatorname{gcd}(m, 4)$ and the odd prime divisors of $m$. We show that $|G| \leq q(m) k^{2} / \varphi(m)$ where $\varphi$ denotes Euler's totient function. This strengthens a recent result of Cocke and Venkataraman. As an application we classify all finite groups with $k<36$. This is motivated by a conjecture of Thompson and unifies several partial results in the literature.
\end{abstract}

Keywords Finite groups $\cdot$ Number of elements $\cdot$ Maximal order

Mathematics Subject Classification 20D60 - 20E34

\section{Introduction}

Let $G$ be a finite group with maximal element order $m:=\max \{|\langle x\rangle|: x \in G\}$. Recently, Cocke and Venkataraman [3] have shown that the order of $G$ is bounded by a function on the number $k$ of elements of order $m$ in $G$. More precisely, $\varphi(m)$ divides $k$ and $|G| \leq m k^{2} / \varphi(m)$ (here and in the following $\varphi$ denotes Euler's totient function). The authors have noticed that their bound is best possible for the Frobenius group $G=C_{p} \rtimes C_{p-1}$ where $p$ is a prime. We have observed conversely that sharpness can only hold if $m$ is neither divisible by 8 nor by the square of an odd prime. In fact, our main theorem gives a much stronger bound on $|G|$. To state it, we introduce the following notation.

Communicated by John S. Wilson.

Benjamin Sambale

sambale@math.uni-hannover.de

Philipp Wellmann

wellmann@stud.uni-hannover.de

1 Institut für Algebra, Zahlentheorie und Diskrete Mathematik, Leibniz Universität Hannover, Welfengarten 1, 30167 Hannover, Germany 
Let $p_{1}, \ldots, p_{r}$ be the distinct prime divisors of a positive integer $n$. We define $q(n):=p_{1} \ldots p_{r}$ if $4 \nmid n$ and $q(n):=2 p_{1} \ldots p_{r}$ if $4 \mid n$ (note that $q(1)=1$, the empty product).

Theorem 1 Let $G$ be a finite group with exactly $k$ elements of maximal order $m$. Then

$$
|G| \leq \frac{q(m) k^{2}}{\varphi(m)}
$$

We remark that Cocke and Venkataraman's theorem has been extended but not strengthened in [1].

In the second part of the paper we enumerate finite groups with $k<36$ by computer calculations. This is motivated by the following conjecture of Thompson: If $G$ and $H$ are finite groups with the same multiset of element orders, then $G$ is solvable if and only if $H$ is solvable. Our classification unifies and completes many partial results in the literature for $k=20,22,24,28,30, \ldots$ (see $[2,4-10,14]$ ). There are exactly 13007 groups with $k<36$, the largest have order 3264 .

The paper is based on the bachelor's thesis of the second author written under the supervision of the first author.

\section{Proof of the main theorem}

Let $x \in G$ be an element of maximal order. In [3], the authors have shown that every coset of $\langle x\rangle$ in $\mathrm{C}_{G}(x)$ contains at least $\varphi(m)$ elements of order $m$. To prove our stronger result, we show more generally that "most" cosets of $\langle x\rangle$ in $\mathrm{N}_{G}(\langle x\rangle)$ contain at least $\varphi(m)$ elements of order $m$.

Proof Let $X:=\langle x\rangle \leq G$ be a cyclic subgroup of maximal order $m$. Each conjugate of $X$ contains exactly $\varphi(m)$ elements of order $m$. Hence, $\left|G: \mathrm{N}_{G}(X)\right| \varphi(m) \leq k$. Let $m=p_{1}^{a_{1}} \ldots p_{r}^{a_{r}}$ be the prime factorization of $m$ where $p_{1}<\ldots<p_{r}$. It is well-known that there is an injective homomorphism

$$
\Gamma: \mathrm{N}_{G}(X) / \mathrm{C}_{G}(X) \rightarrow \operatorname{Aut}(X) \cong(\mathbb{Z} / m \mathbb{Z})^{\times} \cong \underset{i=1}{r}\left(\mathbb{Z} / p_{i}^{a_{i}} \mathbb{Z}\right)^{\times}
$$

For $p_{i}>2$, the group $\left(\mathbb{Z} / p_{i}^{a_{i}} \mathbb{Z}\right)^{\times}$is cyclic of order $\varphi\left(p_{i}^{a_{i}}\right)=(p-1) p_{i}^{a_{i}-1}$ and its Sylow $p$-subgroup is generated by $1+p_{i}+p_{i}^{a_{i}} \mathbb{Z}$. On the other hand, for $p=2$ we have $\left(\mathbb{Z} / 2^{a} \mathbb{Z}\right)^{\times}=\left\langle-1+2^{a} \mathbb{Z}\right\rangle \times\left\langle 5+2^{a} \mathbb{Z}\right\rangle$. If $p_{1}>2$, let $N / C_{G}(X)$ be the preimage of $\times_{i=1}^{r}\left\langle 1+p_{i}+p_{i}^{a_{i}} \mathbb{Z}\right\rangle$ under $\Gamma$. Otherwise, let $N / \mathrm{C}_{G}(X)$ be the preimage of

$$
\left\langle 5+2^{a_{1}} \mathbb{Z}\right\rangle \times \underset{i=2}{\times}\left\langle 1+p_{i}+p_{i}^{a_{i}} \mathbb{Z}\right\rangle .
$$

In either case, $N / \mathrm{C}_{G}(X)$ is cyclic. Moreover, $\left|\mathrm{N}_{G}(X): N\right| \leq\left(p_{1}-1\right) \ldots\left(p_{r}-1\right)$ if $4 \nmid m$, and $\left|\mathrm{N}_{G}(X): N\right| \leq 2\left(p_{1}-1\right) \ldots\left(p_{r}-1\right)$ otherwise. 
We will show that every coset of $X$ in $N$ contains at least $\varphi(m)$ elements of order $m$. It will then follow that $|N: X| \varphi(m) \leq k$ and

$$
|G|=\left|G: \mathrm{N}_{G}(X)\right|\left|\mathrm{N}_{G}(X): N\right||N: X||X| \leq \frac{m k^{2}}{\varphi(m)^{2}}\left|\mathrm{~N}_{G}(X): N\right| \leq \frac{q(m) k^{2}}{\varphi(m)} .
$$

Let $y \in N$ and $x^{y}=y^{-1} x y=x^{s}$ for some $s \in \mathbb{Z}$. We aim to determine integers $\alpha$ such that $y x^{\alpha}$ has order $m$. Let $1 \leq i \leq r$ and let $q:=m / p_{i}$. The choice of $N$ shows that $q(m)$ divides $s-1$. This implies

$$
1+s+\ldots+s^{q-1}=\frac{s^{q}-1}{s-1}=\sum_{l=1}^{q}\left(\begin{array}{l}
q \\
l
\end{array}\right)(s-1)^{l-1} \equiv q \quad(\bmod m) .
$$

It follows that

$$
\left(y x^{\alpha}\right)^{q}=y^{q}\left(x^{\alpha}\right)^{y^{q-1}}\left(x^{\alpha}\right)^{y^{q-2}} \ldots x^{\alpha}=y^{q} x^{\left(s^{q-1}+s^{q-2}+\ldots+1\right) \alpha}=y^{q} x^{q \alpha} .
$$

Note that $y^{q} \in \mathrm{C}_{G}(X)$. It is easy to see that there are at least $\varphi\left(p_{i}^{a_{i}}\right)$ choices for $\alpha\left(\bmod p_{i}^{a_{i}}\right)$ such that the $p_{i}$-factor of $y^{q} x^{q \alpha}$ is non-trivial, i.e. the order of $y x^{\alpha}$ is divisible by $p_{i}^{a_{i}}$. By the Chinese remainder theorem, these choices can be made independently for each $i$. In total we obtain at least $\varphi(m)=\varphi\left(p_{1}^{a_{1}}\right) \ldots \varphi\left(p_{r}^{a_{r}}\right)$ elements of order $m$ in the coset $y X$.

\section{Finite groups with few elements of maximal order}

As before, let $G$ be a finite group with exactly $k$ elements of maximal order $m$. We fix some $X=\langle x\rangle \leq G$ of order $m$. To get more precise results we start by analyzing the proof of Theorem 1 . Suppose that we have equality $|G|=q(m) k^{2} / \varphi(m)$. Then we obtain:

(i) $\left|G: \mathrm{N}_{G}(X)\right|=k / \varphi(m)$, i.e. the cyclic subgroups of order $m$ are conjugate in $G$.

(ii) $\left|\mathrm{N}_{G}(X): N\right|=2^{i}\left(p_{1}-1\right) \ldots\left(p_{r}-1\right)$ (where $i=1$ if $4 \mid m$ and 0 otherwise). In particular, the numbers $p_{i}-1$ divide $|G|$.

(iii) All elements of order $m$ lie in $N$ and every coset of $X$ in $N$ contains exactly $\varphi(m)$ elements of order $m$. Thus, $N \unlhd G$. Let $y \in \mathrm{C}_{G}(X)$. Then $\langle x, y\rangle$ is abelian and there exists $y^{\prime}$ such that $\langle x, y\rangle=\langle x\rangle \times\left\langle y^{\prime}\right\rangle$. Since $y^{\prime} X$ has only $\varphi(m)$ elements of order $m$, the order of $y^{\prime}$ must divide $p_{1}^{a_{1}-1} \ldots p_{r}^{a_{r}-1}$. In particular, $\left|\mathrm{C}_{G}(X): X\right|$ divides $p_{1}^{a_{1}-1} \ldots p_{r}^{a_{r}-1}$. Thus, if $m$ is squarefree (i.e. $a_{1}=\ldots=a_{r}=1$ ), then $\mathrm{C}_{G}(X)=X$ and $m k^{2} / \varphi(m)=|G|=\left|G: \mathrm{C}_{G}(X)\right||X| \leq \mathrm{km}$. Consequently, $k=\varphi(m)$ and $G=\mathrm{N}_{G}(X)$. We extend this observation to the case $q(m)=m$. This characterizes groups with equality in Cocke-Venkataraman's original bound.

Proposition 2 With the notation above, suppose that $|G|=m k^{2} / \varphi(m)$. Then

(i) $m=q(m)$, 
(ii) $k=\varphi(m)$,

(iii) $G$ has a unique cyclic subgroup $X$ of order $m$.

(iv) $\mathrm{C}_{G}(X)=X$ and $G / X \cong \operatorname{Aut}(X)$.

Proof Since $q(m) \leq m$, the claim $m=q(m)$ follows from Theorem 1 . The analysis above implies $\mathrm{C}_{G}(X)=N \unlhd G$ and $|N|=m k / \varphi(m)$. It follows that $\left|G: \mathrm{C}_{G}(X)\right|=k$, i.e. all elements of order $m$ are conjugate in $G$. Hence, for every element $y \in N$ of order $m$ we obtain $\mathrm{C}_{G}(y)=N$. Therefore, $N$ is abelian. The above arguments show further that $N=X \times Y$ where $Y$ is an elementary abelian 2-group. If $4 \nmid m$, then $Y=1$. Now suppose $4 \mid m$ and let $X_{2}$ be the Sylow 2-subgroup of $X$. For every $y \in Y$ there exists $g \in G$ such that $x^{g}=x y$. This yields $g \in \mathrm{C}_{G}\left(x^{2}\right)$. Hence, $H:=\mathrm{C}_{G}\left(x^{2}\right)$ acts on $X_{2} \times Y$ and permutes the elements of order 4 transitively. The number of those elements is $2|Y|$ which in turn must divide $|H / N|$. Let $g \in H \backslash N$ be a 2-element. Then $g$ must be an involution, because otherwise $x^{4} g$ would be an element of order $\geq m$ outside $N$. For any $z \in X_{2} \times Y$ also $g z$ is a 2-element, since $(g z)^{2}=z^{g} z \in X_{2} \times Y$. By the same argument, $g z$ is an involution and therefore $z^{g}=z^{-1}$. But this completely determines the action of $g$ on $X_{2} \times Y$. Since $g \in H=\mathrm{C}_{G}\left(x^{2}\right)$ and $X=\left\langle x^{2}\right\rangle X_{2}$, also the action of $g$ on $X$ is uniquely determined. Consequently, there is only one non-trivial 2-element in $H / N$. In particular, $|H / N|$ is not divisible by 4 . This leads to $Y=1$ and $N=X$. Finally, $m=|X|=m k / \varphi(m)$ yields $k=\varphi(m)$.

For $q(m)=m$ it is easy to show that the holomorph $G:=C_{m} \rtimes \operatorname{Aut}\left(C_{m}\right)$ does indeed have a unique maximal cyclic subgroup of order $m$. Usually, many non-split extensions of $C_{m}$ by $\operatorname{Aut}\left(C_{m}\right)$ fulfill the conditions of Proposition 2 as well. On the other hand, for $m=4$ the quaternion group $Q_{8}$ has six (and not two) elements of order 4.

Since the classification of $p$-groups is particularly complicated we improve Theorem 1 for $p$-groups as follows.

Proposition 3 Let $G$ be a p-group with exactly $k$ elements of order $p^{e}=\exp (G)$. Let $k_{p}$ be the p-part of $k$. Then

$$
|G| \leq \begin{cases}p^{2-e+\left\lfloor\log _{p}(k /(p-1))\right\rfloor} k_{p} & \text { if } p>2, \\ 2^{3-e+\left\lfloor\log _{2}(k)\right\rfloor} k_{2} & \text { if } p=2 .\end{cases}
$$

Moreover, if $e=2$, then $|G| \leq 4 k$ for $p=2$ and $|G| \leq 9 k / 2$ for $p=3$.

Proof The $k / \varphi\left(p^{e}\right)$ cyclic subgroups of order $p^{e}$ of $G$ distribute into orbits under the conjugation action. Since each orbit size divides $|G|$, we find a cyclic subgroup $X$ of order $p^{e}$ such that $\left|G: \mathrm{N}_{G}(X)\right| \leq k_{p} / p^{e-1}$. Let $N \leq \mathrm{N}_{G}(X)$ be as in the proof of Theorem 1. For $p>2$ we have $N=\mathrm{N}_{G}(X)$ and for $p=2$ we have $\left|\mathrm{N}_{G}(X): N\right| \leq 2$. As in the proof of Theorem $1,|N| \leq k p^{e} / \varphi\left(p^{e}\right)=p k /(p-1)$. From Lagrange's Theorem we obtain $|N| \leq p^{1+\left\lfloor\log _{p}(\bar{k} /(p-1))\right\rfloor}$. Now the first claim follows since $|G|=\left|G: \mathrm{N}_{G}(X)\right|\left|\mathrm{N}_{G}(X): N\right||N|$.

Next let $e=2$ and $p \leq 3$. By theorems of Wall [13] and Laffey [11] the number of elements of order $p$ is less than $3|G| / 4$ for $p=2$ and less than $7|G| / 9$ for $p=3$. This implies $|G|-k \leq 3|G| / 4$ and $|G|-k \leq 7|G| / 9$ respectively. The second claim follows. 
For odd $p$, the first bound in Proposition 3 is best possible for cyclic groups and groups of exponent $p$. For $p=2$ however, the bound is optimal for the 2 -groups of maximal nilpotency class, i.e. for the dihedral, semidihedral and quaternion groups. We do not know if there are other groups attaining the bound.

The following lemma is taken from [2, Lemma 8]. For the convenience of the reader we provide a proof.

Lemma 4 With the notation above there exists an integer a such that $|G|$ divides $\mathrm{km}^{a}$.

Proof Let $p$ be a prime divisor of $|G|$ which does not divide $m$. Then $p$ does not divide $\left|\mathrm{C}_{G}(x)\right|$ either, since otherwise there would be an element of order $p m$. Hence, $p$ divides the size $\left|G: \mathrm{C}_{G}(x)\right|$ of the conjugacy class of $x$. The union of all those conjugacy classes is the set of elements of order $m$. Therefore, $p$ divides $k$.

The bound in Theorem 1 is often large when $m$ is small. The next observation excludes many exceptional cases.

Lemma 5 With the notation above let $m=2 p$ where $p$ is an odd prime. Then $|G| \leq$ $2 k(k+1)$.

Proof For $y \in \mathrm{C}_{G}(X) \backslash X$ we have $\langle x, y\rangle \leq C_{2 p} \times C_{2 p}$. So there are at least $p$ elements of order $2 p$ in the coset $y X$. It follows that $\frac{1}{2}\left|\mathrm{C}_{G}(X)\right|-1 \leq k$ and $|G|=$ $\left|G: \mathrm{C}_{G}(X)\right|\left|\mathrm{C}_{G}(X)\right| \leq 2 k(k+1)$.

Proposition 6 If $|G| \in\left\{2^{9}, 2^{10}, 2^{9} \cdot 3\right\}$, then $k \geq 36$.

Proof Suppose that $k<36$. First we consider $|G|=2^{9}$. Any element $x \in G$ of maximal order $m$ must lie in some maximal subgroup $M<G$ (otherwise $G$ is cyclic and $k=2^{8}>35$ ). By making use of the small groups library in GAP [12], it turns out that $M$ has exactly 32 elements of order $m$ (there are no groups with fewer elements of maximal order). Moreover, there are just 89 candidates for $M$ up to isomorphism. With the grpconst package in GAP we show that none of those extends to a group of order $2^{9}$ with $k<36$. By an inductive argument there are no groups of order $2^{10}$ with $k<36$.

Now let $|G|=2^{9} \cdot 3$. Here $m$ is divisible by 3 , since otherwise we get examples of order $2^{9}$. Hence, $X$ lies in the centralizer of a Sylow 3-subgroup $Q$ of $G$. In almost all cases, $Q$ is normal in $G$ and $\left|G: \mathrm{C}_{G}(Q)\right| \leq 2$. However, a Sylow 2-subgroup of $\mathrm{C}_{G}(Q)$ has at least 32 elements of maximal order (see above). Therefore, $\mathrm{C}_{G}(Q)$ has at least 64 elements of maximal order. Consequently, $Q$ is not normal in $G$. By consulting the Smal IGroups Information command in GAP, we learn that there are "only" 114,464 such groups which can be checked in a matter of hours.

Theorem 7 There are exactly 13007 finite groups with $k<36$ elements of maximal order m. Just 10684 of those occur for $k=32$. The distribution of the group orders for $k \neq 32$ is given in the appendix.

Proof If $k$ is odd, so is $\varphi(m)$ and it follows that $m \in\{1,2\}$. It is well-known that $G$ must be an elementary abelian 2-group in this situation. In particular, $|G|=1=k$ or $k=|G|-1=2^{n}-1$ for $1 \leq n \leq 5$. Therefore, we may restrict ourselves to even 
values of $k$. It is straight forward to determine (by computer) for each $k$ the possible integers $m$ such that $\varphi(m)$ divides $k$. If $k<24$, then Theorem 1 yields $|G| \leq 2000$. These groups can be enumerated quickly with GAP [12] by taking Proposition 6 into account. It is perhaps surprising that there are no groups with $k=34$.

For the remainder of the proof we focus on parameters where $|G|>2000$. If $k$ does not divide $|G| / m$, then there are at least two conjugacy classes of elements of order $m$. Hence, we may choose $X$ such that $\left|G: \mathrm{C}_{G}(X)\right| \leq k / 2$. Together with the coset counting argument $\left|\mathrm{C}_{G}(X)\right| \leq m k / \varphi(m)$ from the proof of Theorem 1, many cases can be excluded in an automatic fashion. Moreover, we use the results obtained above without comment. The remaining cases are handled by ad hoc arguments:

(i) $k=24, m=84=2^{2} \cdot 3 \cdot 7$ and $|G|=m k^{2} / \varphi(m)=2016$. Here the structure of $G$ is fairly restricted by Proposition 2. In particular, the unique $X$ is contained in a subgroup $H \leq G$ of index 2 . Since we have already determined all candidates for $H$, the possible extensions $G$ can be obtained with the grpconst package in GAP. It turns out that there are exactly 32 such groups of order 2016.

(ii) $k=28, m=12$ and $|G|=2016=2^{5} \cdot 3^{2} \cdot 7$. It is clear that $\left|\mathrm{C}_{G}(\mathrm{X})\right|$ is not divisible by 7. Suppose first that there exists some $X$ such that $\left|G: \mathrm{C}_{G}(X)\right|=k$. It can be checked by GAP that $\mathrm{C}_{G}(X)$ is isomorphic to $C_{12} \times C_{6}$ or to $C_{12} \times S_{3}$. However, the former group already contains 32 elements of order 12 . Thus, $\mathrm{C}_{G}(X) \cong C_{12} \times S_{3}$ and all 28 elements of order 12 lie in $\mathrm{C}_{G}(X)$. Since $\mathrm{C}_{G}(X)$ is generated by those elements, it follows that $\mathrm{C}_{G}(X) \unlhd G$. However, $X=\mathrm{Z}\left(\mathrm{C}_{G}(X)\right)$ is characteristic in $\mathrm{C}_{G}(\mathrm{X})$, so the generators of $X$ are not conjugate to elements outside $X$. This contradiction implies that we find $X$ with $\left|G: \mathrm{C}_{G}(X)\right| \leq 14$. Coset counting gives $\left|\mathrm{C}_{G}(X)\right| \leq 3 k$ and $|G| \leq 42 k=1176$, a contradiction.

(iii) $k=32, m=12$ and $|G|=2304=2^{8} \cdot 3^{2}$. Since $\left|\mathrm{C}_{G}(X)\right| \leq m k / \varphi(m)=96$, we must have $\left|G: \mathrm{C}_{G}(X)\right|=k$ and $\left|\mathrm{C}_{G}(\mathrm{X})\right|=72$. As in the previous case, $\mathrm{C}_{G}(X) \cong$ $C_{12} \times C_{6}$ or $\mathrm{C}_{G}(X) \cong C_{12} \times S_{3}$. Suppose first that $\mathrm{C}_{G}(X) \cong C_{12} \times C_{6}$. Then $\mathrm{C}_{G}(X) \unlhd G$ and there exists a subgroup $\mathrm{C}_{G}(X) \leq H \leq G$ such that $|G: H|=2$. There are 81 candidates for $H$ (with $(k, m)=(32,12)$ ), but none of those can be extended to a group of order $2^{8} \cdot 3^{2}$ with $k=32$. Next let $\mathrm{C}_{G}(X)=C_{12} \times S_{3}$. Here $\mathrm{C}_{G}(X)$ contains only 24 elements of order $m$. Hence, $X$ is conjugate to some subgroup $Y$ outside $\mathrm{C}_{G}(X)$. Moreover, $\mathrm{C}_{G}(X)$ and $\mathrm{C}_{G}(Y)$ have at least 16 elements of order $m$ in common. This yields $\mathrm{C}_{G}(X) \cap C_{G}(Y) \cong C_{12} \times C_{3}$. It follows easily that $G$ has a normal Sylow 3-subgroup. Again there must be some $\mathrm{C}_{G}(X) \leq H \leq G$ with $|G: H|=2$. This was already dismissed above.

(iv) $k=32, m=30$ and $|G|=2880=2^{6} \cdot 3^{2} \cdot 5$. Since $\left|\mathrm{C}_{G}(X)\right| \leq m k / \varphi(m)=120$, we must have $\left|G: \mathrm{C}_{G}(X)\right|=k$ and $\mathrm{C}_{G}(X) \cong C_{30} \times C_{3}$. Hence, all elements of order $m$ lie in $\mathrm{C}_{G}(X)$ and $\mathrm{C}_{G}(X) \unlhd G$. Consequently, there exists $\mathrm{C}_{G}(X) \leq H \leq G$ with $|G: H|=2$. However, there are no candidates for $H$.

(v) $k=32, m=68=2^{2} \cdot 17$ and $|G|=2176=2^{7} \cdot 17=m k^{2} / \varphi(m)$. Here the structure of $G$ is determined by Proposition 2. As in the first case, the desired groups can be constructed explicitly. There are 8 of them.

(vi) $k=32, m=102=2 \cdot 3 \cdot 17$ and $|G|=3264=2^{6} \cdot 3 \cdot 17=m k^{2} / \varphi(m)$. Again Proposition 2 applies and there are just 4 such groups. 
The following corollary implies a special case of Thompson's Conjecture mentioned in the introduction (cf. [2, Proposition 1]).

Corollary 8 Let $G$ and $H$ be finite groups of the same order and the same maximal element order $m$. Suppose that $G$ and $H$ both have exactly $k<36$ elements of order $m$. Then $G$ is solvable if and only if $H$ is solvable.

Proof Since we have computed the groups in Theorem 7 explicitly, it is easy to extract the non-solvable ones:

- $(k, m)=(20,6): G=S_{5}$,

- $(k, m)=(24,5): G=A_{5}$,

- $(k, m)=(24,10): G=A_{5} \times C_{2}, \operatorname{SL}(2,5), S_{5} \times C_{2}$ and $\operatorname{SL}(2,5) \rtimes C_{2}$.

The claim now follows by inspection of the table in the appendix.

Acknowledgements The first author is supported by the German Research Foundation (SA 2864/1-2 and SA 2864/3-1).

Funding Open Access funding enabled and organized by Projekt DEAL.

Open Access This article is licensed under a Creative Commons Attribution 4.0 International License, which permits use, sharing, adaptation, distribution and reproduction in any medium or format, as long as you give appropriate credit to the original author(s) and the source, provide a link to the Creative Commons licence, and indicate if changes were made. The images or other third party material in this article are included in the article's Creative Commons licence, unless indicated otherwise in a credit line to the material. If material is not included in the article's Creative Commons licence and your intended use is not permitted by statutory regulation or exceeds the permitted use, you will need to obtain permission directly from the copyright holder. To view a copy of this licence, visit http://creativecommons.org/licenses/by/4.0/.

\section{Appendix}

The following table contains the parameters of groups $G$ with exactly $k$ elements of maximal order $m$ where $k<36$ and $k \neq 32$. An entry of the form $n^{s}$ means that there are $s$ groups of order $n$ up to isomorphism. The small group ids (if available) of all groups including $k=32$ can be accessed on the first author's homepage.

\begin{tabular}{llll}
\hline$k$ & $m$ & $|G|^{\#}$ & Total \\
\hline 1 & 1 & 1 & 1 \\
& 2 & 2 & 1 \\
& 3 & 3,6 & 2 \\
& 4 & 4,8 & 2 \\
3 & 6 & $6,12^{2}$ & 3 \\
\hline
\end{tabular}




\begin{tabular}{|c|c|c|c|}
\hline$k$ & $m$ & $|G|^{\#}$ & Total \\
\hline \multirow[t]{5}{*}{4} & 4 & 8,16 & 2 \\
\hline & 5 & $5,10,20$ & 3 \\
\hline & 8 & $8,16^{3}$ & 4 \\
\hline & 10 & $10,20^{2}, 40^{2}$ & 5 \\
\hline & 12 & $12,24^{5}, 48^{4}$ & 10 \\
\hline \multirow[t]{6}{*}{6} & 4 & 8,24 & 2 \\
\hline & 6 & $12,18,24^{3}, 36,72$ & 7 \\
\hline & 7 & $7,14,21,42$ & 4 \\
\hline & 9 & 9,18 & 2 \\
\hline & 14 & $14,28^{2}, 42,84^{2}$ & 6 \\
\hline & 18 & $18,36^{2}$ & 3 \\
\hline 7 & 2 & 8 & 1 \\
\hline \multirow[t]{10}{*}{8} & 3 & $9,12,18$ & 3 \\
\hline & 4 & $16^{3}, 32$ & 4 \\
\hline & 6 & $18,24^{2}, 36^{2}, 48^{2}, 72,144^{2}$ & 10 \\
\hline & 8 & $16^{2}, 32^{11}$ & 13 \\
\hline & 12 & $24,48^{11}, 96^{16}$ & 28 \\
\hline & 15 & $15,30^{3}, 60^{3}, 120$ & 8 \\
\hline & 16 & $16,32^{3}$ & 4 \\
\hline & 20 & $20,40^{5}, 80^{9}, 160^{4}$ & 19 \\
\hline & 24 & $24,48^{9}, 96^{14}$ & 24 \\
\hline & 30 & $30,60^{6}, 120^{12}, 240^{8}$ & 27 \\
\hline \multirow[t]{2}{*}{10} & 11 & $11,22,55,110$ & 4 \\
\hline & 22 & $22,44^{2}, 110,220^{2}$ & 6 \\
\hline \multirow[t]{11}{*}{12} & 4 & $16^{3}, 32^{3}, 48$ & 7 \\
\hline & 6 & 36 & 1 \\
\hline & 8 & $48^{2}$ & 2 \\
\hline & 10 & $20,40^{3}, 80^{4}$ & 8 \\
\hline & 12 & $24,36,48^{4}, 72^{5}, 144^{5}$ & 16 \\
\hline & 13 & $13,26,39,52,78,156$ & 6 \\
\hline & 21 & $21,42^{3}, 63^{2}, 84,126^{4}, 252$ & 12 \\
\hline & 26 & $26,52^{2}, 78,104^{2}, 156^{2}, 312^{2}$ & 10 \\
\hline & 28 & $28,56^{5}, 84,112^{4}, 168^{5}, 336^{4}$ & 20 \\
\hline & 36 & $36,72^{5}, 144^{4}$ & 10 \\
\hline & 42 & $42,84^{6}, 126^{2}, 168^{8}, 252^{8}, 504^{8}$ & 33 \\
\hline 14 & 6 & $24,48^{4}$ & 5 \\
\hline 15 & 2 & 16 & 1 \\
\hline
\end{tabular}




\begin{tabular}{|c|c|c|c|}
\hline$k$ & $m$ & $|G|^{\#}$ & Total \\
\hline \multirow[t]{12}{*}{16} & 4 & $32^{4}, 64$ & 5 \\
\hline & 8 & $32^{9}, 64^{85}, 128^{9}$ & 103 \\
\hline & 12 & $36,48^{5}, 72^{6}, 96^{47}, 144^{10}, 192^{36}, 288^{14}, 576^{11}$ & 130 \\
\hline & 16 & $32^{2}, 64^{16}$ & 18 \\
\hline & 17 & $17,34,68,136,272$ & 5 \\
\hline & 20 & $40,80^{11}, 160^{32}, 320^{25}$ & 69 \\
\hline & 24 & $48^{2}, 96^{37}, 192^{210}$ & 249 \\
\hline & 32 & $32,64^{3}$ & 4 \\
\hline & 34 & $34,68^{2}, 136^{2}, 272^{2}, 544^{2}$ & 9 \\
\hline & 40 & $40,80^{9}, 160^{23}, 320^{20}$ & 53 \\
\hline & 48 & $48,96^{9}, 192^{14}$ & 24 \\
\hline & 60 & $60,120^{13}, 240^{54}, 480^{76}, 960^{32}$ & 176 \\
\hline \multirow[t]{9}{*}{18} & 4 & 36 & 1 \\
\hline & 6 & $54^{3}, 108$ & 4 \\
\hline & 9 & $27^{2}, 54^{3}, 81,162^{2}$ & 8 \\
\hline & 14 & $28,56^{3}, 84^{2}, 168^{4}$ & 10 \\
\hline & 18 & $36,54^{3}, 72^{3}, 108^{9}, 162,216^{3}, 324^{4}$ & 24 \\
\hline & 19 & $19,38,57,114,171,342$ & 6 \\
\hline & 27 & 27,54 & 2 \\
\hline & 38 & $38,76^{2}, 114,228^{2}, 342,684^{2}$ & 9 \\
\hline & 54 & $54,108^{2}$ & 3 \\
\hline \multirow[t]{8}{*}{20} & 4 & $32^{5}$ & 5 \\
\hline & 6 & 36,120 & 2 \\
\hline & 10 & 50,100 & 2 \\
\hline & 25 & $25,50,100$ & 3 \\
\hline & 33 & $33,66^{3}, 132,165,330^{3}, 660$ & 10 \\
\hline & 44 & $44,88^{5}, 176^{4}, 220,440^{5}, 880^{4}$ & 20 \\
\hline & 50 & $50,100^{2}, 200^{2}$ & 5 \\
\hline & 66 & $66,132^{6}, 264^{8}, 330,660^{6}, 1320^{8}$ & 30 \\
\hline \multirow[t]{2}{*}{22} & 23 & $23,46,253,506$ & 4 \\
\hline & 46 & $46,92^{2}, 506,1012^{2}$ & 6 \\
\hline \multirow[t]{5}{*}{24} & 4 & $32^{7}, 64^{3}$ & 10 \\
\hline & 5 & $25,50,60,75,100^{2}, 200$ & 7 \\
\hline & 6 & $36,48^{2}, 54,72^{4}, 96^{3}, 108^{2}, 144^{2}, 216,288^{4}$ & 20 \\
\hline & 8 & $32,96^{5}$ & 6 \\
\hline & 9 & 36,72 & 2 \\
\hline
\end{tabular}




\begin{tabular}{|c|c|c|c|}
\hline$k$ & $m$ & $|G|^{\#}$ & Total \\
\hline & 10 & $50,100^{2}, 120^{2}, 150,200^{4}, 240^{2}, 300,400^{3}, 600,1200$ & 18 \\
\hline & 12 & $48^{3}, 72^{3}, 96^{44}, 144^{12}, 192^{38}, 288^{19}, 576^{14}$ & 133 \\
\hline & 18 & $72^{2}, 144^{4}$ & 6 \\
\hline & 20 & $40,80^{4}, 120,160^{4}, 240,480$ & 12 \\
\hline & 24 & $72,144^{12}, 288^{24}$ & 37 \\
\hline & 28 & $56,112^{11}, 168,224^{16}, 336^{11}, 672^{16}$ & 56 \\
\hline & 30 & $60,90,120^{9}, 180^{3}, 240^{29}, 360^{7}, 480^{27}, 720^{6}, 1440^{2}$ & 85 \\
\hline & 35 & $35,70^{3}, 105,140^{3}, 210^{3}, 280,420^{3}, 840$ & 16 \\
\hline & 36 & $72,144^{11}, 288^{16}$ & 28 \\
\hline & 39 & $39,78^{3}, 117^{2}, 156^{3}, 234^{4}, 312,468^{4}, 936$ & 19 \\
\hline & 45 & $45,90^{3}, 180^{3}, 360$ & 8 \\
\hline & 52 & $52,104^{5}, 156,208^{9}, 312^{5}, 416^{4}, 624^{9}, 1248^{4}$ & 38 \\
\hline & 56 & $56,112^{9}, 168,224^{14}, 336^{9}, 672^{14}$ & 48 \\
\hline & 70 & $70,140^{6}, 210,280^{12}, 420^{6}, 560^{8}, 840^{12}, 1680^{8}$ & 54 \\
\hline & 72 & $72,144^{9}, 288^{14}$ & 24 \\
\hline & 78 & $78,156^{6}, 234^{2}, 312^{12}, 468^{8}, 624^{8}, 936^{14}, 1872^{8}$ & 59 \\
\hline & 84 & $84,168^{13}, 252^{2}, 336^{44}, 504^{18}, 672^{32}, 1008^{48}, 2016^{32}$ & 190 \\
\hline & 90 & $90,180^{6}, 360^{12}, 720^{8}$ & 27 \\
\hline \multirow[t]{2}{*}{26} & 3 & $27^{2}, 54$ & 3 \\
\hline & 6 & $54^{2}, 108^{2}$ & 4 \\
\hline \multirow[t]{5}{*}{28} & 4 & $32^{3}, 64$ & 4 \\
\hline & 10 & $40,80^{4}, 160^{7}$ & 12 \\
\hline & 12 & $72,144^{3}$ & 4 \\
\hline & 29 & $29,58,116,203,406,812$ & 6 \\
\hline & 58 & $58,116^{2}, 232^{2}, 406,812^{2}, 1624^{2}$ & 10 \\
\hline \multirow[t]{4}{*}{30} & 6 & $48,72,96^{5}, 144,288$ & 9 \\
\hline & 22 & $44,88^{3}, 220,440^{3}$ & 8 \\
\hline & 31 & $31,62,93,155,186,310,465,930$ & 8 \\
\hline & 62 & $62,124^{2}, 186,310,372^{2}, 620^{2}, 930,1860^{2}$ & 12 \\
\hline 31 & 2 & 32 & 1 \\
\hline
\end{tabular}




\section{References}

1. Benesh, B., Cocke, W.: On the number of elements in a group whose order is divisible by a common natural number. Math. Proc. R. Ir. Acad. 120A, 1-5 (2020)

2. Chen, G., Shi, W.: Finite groups with 30 elements of maximal order. Appl. Categ. Struct. 16, 239-247 (2008)

3. Cocke, W., Venkataraman, G.: On the number of elements of maximal order in a group. Am. Math. Mon. 126, 66-69 (2019)

4. Han, Z., Song, R.: Finite groups having exactly 22 elements of maximal order. Int. J. Algebra 8, 353-355 (2014)

5. Han, Z., Song, R.: Finite groups having exactly 28 elements of maximal order. Int. J. Algebra 8, 563-568 (2014)

6. Han, Z., Song, R.: Finite groups having exactly 44 elements of maximal order. Adv. Math. (China) 45, 61-66 (2016)

7. Han, Z., Xie, L., Guo, P.: Finite groups which have 20 elements of maximal order. Math. Probl. Eng. 2020, 5 (2020)

8. Han, Z., Zhang, L.: Finite groups having exactly 42 elements of maximal order. Ital. J. Pure Appl. Math. 351-354,(2017)

9. Jiang, Q., Shao, C.: Finite groups with 24 elements of maximal order. Front. Math. China 5, 665-678 (2010)

10. Jiang, Y.Y.: Finite groups with 44 or 52 maximal-order elements. J. Hebei Univ. Nat. Sci. 24, 113-116 (2004)

11. Laffey, T.J.: The number of solutions of $x^{3}=1$ in a 3-group. Math. Z. 149, 43-45 (1976)

12. The GAP Group, GAP-Groups, Algorithms, and Programming, Version 4.11.0 (2020). http://www. gap-system.org

13. Wall, C.T.C.: On groups consisting mostly of involutions. Proc. Camb. Philos. Soc. 67, 251-262 (1970)

14. Yang, C.: Finite groups with various numbers of elements of maximum order. Chin. Ann. Math. Ser. A 14, 561-567 (1993)

Publisher's Note Springer Nature remains neutral with regard to jurisdictional claims in published maps and institutional affiliations. 